\section{A Dynamic Model of the Mitochondrial Protein Import Machinery}

\author{
Nikolaus Pfanner, Joachim Rassow, Ida J. van der Klei, \\ and Walter Neupert \\ Institut für Physiologische Chemie \\ Universität München \\ W.8000 München 2 \\ Germany
}

Many proteins are translocated into or across two membranes in order to reach their functional destination; these include many nuclear-encoded mitochondrial and chloroplast proteins, as well as proteins transported into or across the outer membrane of gram-negative bacteria. In eukaryotes, mechanistic insights have been obtained mainly with the mitochondrial two-membrane transport system. By generating translocation intermediates that span both mitochondrial membranes at the same time, it has been demonstrated that the outer and inner membrane translocation machineries cooperate in the import of preproteins (Hartl and Neupert, 1990; Baker and Schatz, 1991). Translocation contact sites were defined as mitochondrial import sites where the outer and inner membranes are so close together that they can be spanned by a single polypeptide chain (Schleyer and Neupert, 1985). On the other hand, the outer and inner membranes each contain a translocation machinery (e.g., receptors, translocation pores) that can act independently of the other (Pfanner and Neupert, 1987; Glick et al., 1991; Hwang et al., 1991; Rassow and Pfanner, 1991).

The most intriguing questions concerning the mitochondrial two-membrane transport system are how the transport machineries are distributed over the two membranes and how they cooperate. This involves the problems of how the machineries of the inner and outer membranes find each other, and how the membranes are organized to allow a productive interaction. Furthermore, it is important to know how the two translocation systems are arranged to allow translocation of proteins to the inner membrane or matrix without intermittent release of the precursors into the intermembrane space. Here we consider the function and distribution of the mitochondrial translocation machinery under the conditions prevailing in the intact cell. Stimulated by several recent findings, we present a dynamic model of protein translocation across both mitochondrial membranes. This concept puts particular emphasis on the subcompartmentation of the inner mitochondrial membrane and its relation to the outer membrane. It provides also an interpretation for previous findings that could not be explained by the current static models of the mitochondrial import apparatus.

Mitochondrial Inner and Outer Boundary Membranes The organization of the mitochondrial inner membrane is of particular importance. Two regions of the inner membrane can be distinguished in electron micrographs of mitochondria as they appear in intact cells (isotonic, orthodox conditions) (Figure 1): those parts that are in proximity to the outer membrane (the inner boundary membrane) and
Minireview

the remaining parts that form the cristae. Limited areas of the outer and inner boundary membranes stay in such stable contact that they are not separated by mechanical forces (such as those generated by swelling or shrinking of mitochondria) or after removal of the bulk of the outer membrane with detergent. These membrane adhesion sites, or morphological contact sites/areas, comprise about $5 \%-10 \%$ of the outer membrane surface. In membrane adhesion sites, outer and inner membranes are not in direct contact but are separated by a space of about 4-6 $\mathrm{nm}$ (Rassow et al., 1989). Under physiological conditions, however, much larger areas of the outer and inner boundary membranes are in close proximity; more than $90 \%$ of the outer membrane is within about $2-8 \mathrm{~nm}$ of the inner boundary membrane.

\section{Organization of the Import Machinery} in the Mitochondrial Outer Membrane

The close proximity of both boundary membranes may thus allow the formation of translocation contact sites practically all over the outer membrane. In fact, the outer membrane import receptors and the general insertion protein ("pore"), which mediate the insertion of preproteins into the outer membrane, are distributed all over the outer membrane and are not confined to the membrane adhesion sites (Söllner et al., 1989, 1990; Vestweber et al., 1989; Kiebler et al., 1990). Receptors and general insertion protein can assemble with each other and disassemble, indicating a dynamic nature of the transport apparatus (Kiebler et al., 1990).

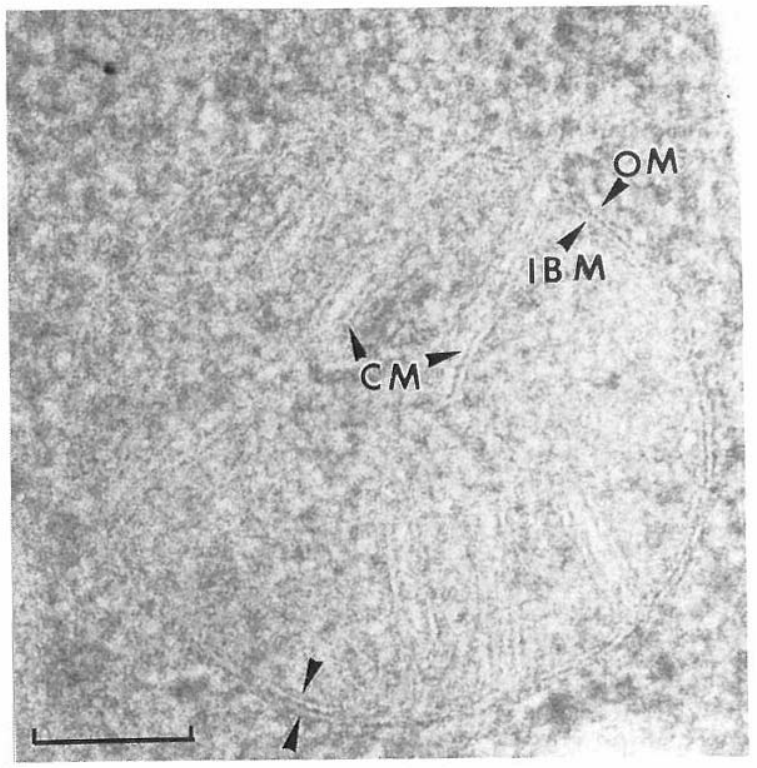

Figure 1. Section through a Yeast Mitochondrion

The outer membrane (OM) and the inner boundary membrane (IBM) are in close proximity. $\mathrm{CM}$, cristae membranes. Sections were cut after freeze substitution of intact Saccharomyces cerevisiae cells. Bar represents $0.1 \mu \mathrm{m}$. Courtesy of Dr. M. Veenhuis, University of Groningen, The Netherlands. 
The outer membrane machinery can completely translocate preproteins across the membrane without involvement of the inner membrane machinery. This is demonstrated by the lack of a requirement for a membrane potential $\Delta \psi$ across the inner membrane for preprotein translocation across the outer membrane, whereas the initial insertion of preproteins into the inner membrane strictly depends on $\Delta \psi$. For example, the precursor of cytochrome $c$ heme lyase employs receptor and general insertion protein (as the other preproteins do) but does not require a $\Delta \psi$ to reach its functional destination in the intermembrane space (Lill et al., 1992); the precursor of the ADPIATP carrier is also translocated to the intermembrane space independently of a $\Delta \psi$, before it enters the inner membrane.

\section{Import Intermediates Exposed \\ to the Intermembrane Space}

When the precursor of ADP/ATP carrier is inserted into the site of the general insertion protein in the absence of $\Delta \psi$, it is protected against proteases added to the outside of mitochondria (Pfanner and Neupert, 1987); after opening of the intermembrane space, the precursor remains associated with the outer membrane, but is now protease sensitive, indicating that major portions of the preprotein are exposed to the intermembrane space (Rassow and Pfanner, 1991).

Preproteins that are translocated to the inner membrane carry positively charged signal sequences. $\Delta \psi$, which is negative on the matrix side, triggers the insertion of the signal sequences into the inner membrane, possibly via an electrophoretic effect (Martin et al., 1991). Preproteins that are in transit across the outer membrane and that possess these signal sequences are committed to engaging the inner membrane import machinery. This can occur while a portion of the preprotein is still associated with the outer membrane, thus yielding a translocation intermediate that spans across both membranes at translocation sites. Transiocation intermediates can also be accumulated after the preprotein has traversed the outer membrane, when the carboxy-terminal portion of the preprotein is exposed to the intermembrane space and the aminoterminal portion has already translocated across the inner membrane (Rassow and Pfanner, 1991; Hwang et al., 1991).

None of the preproteins en route to the inner membrane is freely soluble in the intermembrane space. indicating that transfer through the intermembrane space is coupled to translocation across the membranes, i.e., it occurs at translocation contact sites. The example of cytochrome $c$ heme lyase, however, underscores the functional independence of the outer membrane machinery.

The Import Machinery in the Mitochondrial

\section{Inner Membrane}

Some preproteins can be directly translocated across the inner membrane in mitochondria with a disrupted outer membrane (Hwang et al., 1989), demonstrating a functional independence of the inner membrane import machinery as well. Such transport possesses the same basic characteristics as transport across the inner membrane that occurs at translocation contact sites, including re- quirement for $\Delta \psi$ and a positively charged signal sequence. Most interestingly, the inner membrane transport machinery is present in considerably higher numbers than the outer membrane machinery. Saturation of the outer membrane machinery and translocation contact sites with preproteins spanning both membranes results in the complete blockage of protein import into intact mitochondria. Upon disruption of the outer membrane, large numbers of unoccupied inner membrane machinery become active for direct import of preproteins (Hwang et al., 1989).

\section{Dynamic Model}

These observations suggest that the translocation machinery (pores) in the outer and inner membranes does not form a permanently sealed, continuous channel and that portions of preproteins can be exposed to the intermembrane space. It has to be emphasized, however, that preproteins in transit usually engage both pores at the same time. These sites of translocation do not seem to be restricted to membrane adhesion sites.

We propose the following model for the organization of the mitochondrial protein import apparatus (Figure 2). The import machinery is laterally mobile in the plane of the boundary membranes, while the adhesion sites are stabilized by structural components. A preprotein passing through the outer membrane contacts the aqueous intermembrane space and, driven by $\Delta \psi$, engages the inner membrane import machinery. Putative chaperones between the boundary membranes may facilitate the ordered transfer of the polypeptide into or through the proteincontaining intermembrane space and transiently connect the import machinery of the outer and inner membranes. In this model, an intermembrane-space component that had previously been shown to be necessary for preprotein transport (Schwaiger et al., 1987) may be directly involved in the translocation reaction.

This model implies a dynamic behavior of the import machinery within each membrane and a dynamic interaction between the machineries of the outer and inner membranes. The membrane adhesion sites serve to stabilize mitochondrial structure and provide an exact spacing between the outer and inner membrane machineries, such that the formation of translocation contact sites is facilitated at or near the adhesion sites. Moreover, membrane adhesion sites that form stripes may act as barriers that could prevent diffusion of the import machinery to the cristae membrane. As the number of translocation sites in the inner membrane appears to be greater than those in the outer membrane, signal sequences arriving in the intermembrane space should (possibly with the help of a chaperone) easily find a translocation machinery in the inner membrane.

The possible presence of translocation contact sites throughout the boundary membranes is also supported by previous results showing that ribosomes containing nascent polypeptide chains (assumed to be inserted into translocation contact sites) are preferentially associated with those parts of the outer membrane in close proximity to the inner membrane (Kellems et al., 1975). Using condensed mitochondria with a shrunk matrix and inner membrane, it appeared that protein import occurred at the mor- 
A

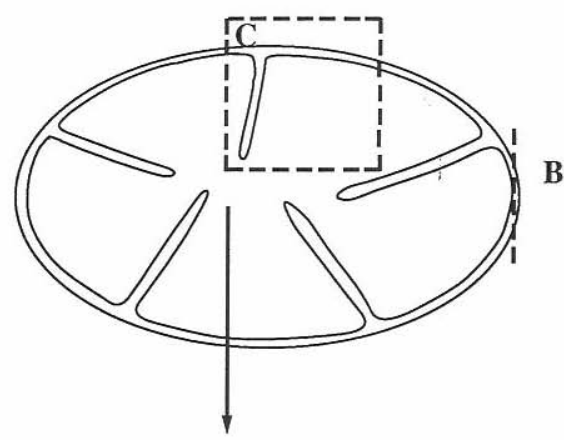

B Aerial view

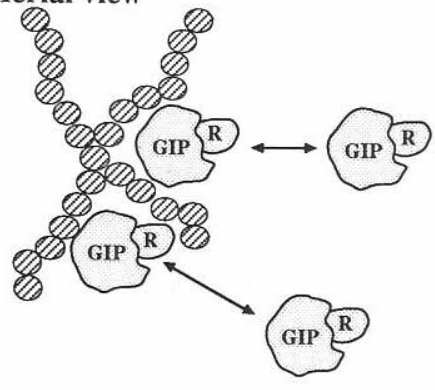

C

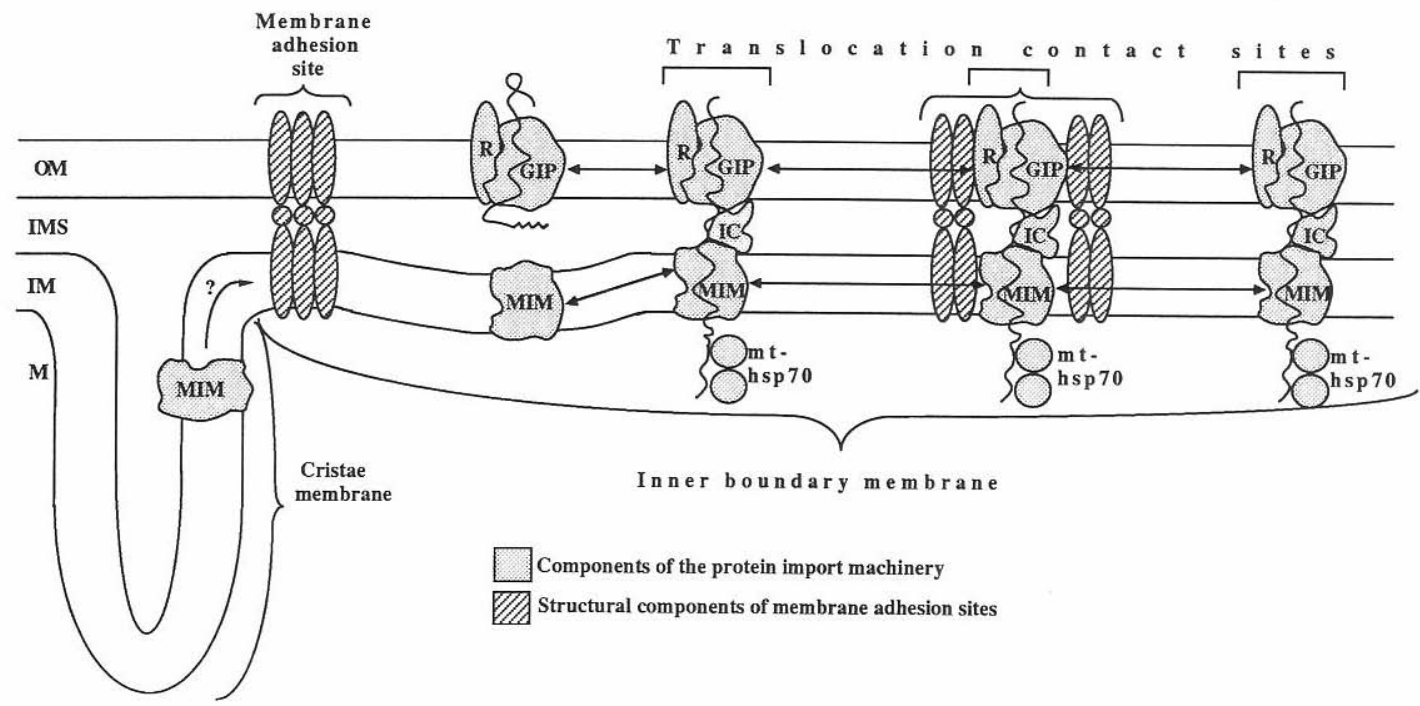

Figure 2. Dynamic Model of Mitochondrial Translocation Contact Sites

GIP, general insertion protein; R, receptor; IC, intermembrane-space component ("chaperone"); IM, inner membrane; IMS, intermembrane space; M, matrix; MIM, mitochondrial inner membrane import machinery; mt-hsp70, heat shock protein of $70 \mathrm{kd}$ in the mitochondrial matrix; OM, outer membrane.

phological contact sites or membrane adhesion sites. Under isotonic conditions, however, the ribosomes were found all over the outer membrane, just as in the case of receptors and general insertion protein. This is in full agreement with our dynamic model that translocation contact sites can be formed wherever outer and inner membranes are in close proximity. In line with this, the frequency of contact sites observed hy electron microscopic techniques seems to depend on the metabolic state of mitochondria (Hackenbrock, 1968; Van Venetie and Verkleij, 1982; Brdiczka et al., 1990).

\section{Relation to Protein Secretion in Bacteria}

How does mitochondrial protein import relate to protein export across two membranes in gram-negative bacteria? The general pathway in bacteria involves distinct translocation steps across plasma membrane and outer membrane, including periplasmic intermediates (Filloux et al., 1990; Sen and Nikaido, 1990; Pugsley et al., 1991). However, the importance of membrane adhesion sites has been a matter of discussion for some 25 years (Bayer, 1981), and at least one exoprotein seems to be translocated across both membranes in a single step, thereby by-passing the periplasm (Koronakis et al., 1991). The existence of a distinct translocation machinery in each membrane, which is not obligatorily coupled to the other, seems to be a common principle of mitochondrial protein import and the general secretion pathway of gram-negative bacteria. So far, however, no sequence homology has been found between components identified in the two transport pathways. One should keep in mind that the mitochondrial and bacterial pathways operate in opposite directions with regard to the evolutionarily related membranes (the mitochondrial inner membrane being derived from a prokaryotic plasma membrane) and use quite different signal sequences. In fact, the export of proteins from the mitochondrial matrix to the intermembrane space and the translocation across the bacterial plasma membrane into the periplasm, which proceed in the same direction, have many similarities, including similar signal sequences and components (Hartl and Neupert, 1990; Baker and Schatz, 1991)

\section{Conclusions}

The mitochondrial outer and inner membranes contain independent machinery for the import of preproteins. These 
import sites are not only active in the membrane adhesion sites that are inseparable by mechanical forces (about $5 \%-10 \%$ of the outer membrane surface) but also in the outer and inner boundary membranes that are in close proximity in mitochondria in vivo $(>90 \%$ of the outer membrane surface). The outer and inner membrane machineries usually cooperate at sites of translocation, probably with the aid of intermembrane space-components that facilitate the transfer of preproteins across the space between both boundary membranes.

\section{References}

Baker, K. P., and Schatz, G. (1991). Nature 349, 205-208

Bayer, M. E. (1981). Int. Rev. Cytol. (Suppl.) 12, 39-70.

Brdiczka, D., Bücheler, K., Kottke, M., Adams, V., and Nalam, V. K. (1990). Biochim. Biophys. Acta 1018, 234-238.

Filloux, A., Bally, M., Ball, G., Akrim, M., Tommassen, J., and Lazdunski, A. (1990). EMBO J. 9, 4323-4329.

Glick, B., Wachter, C., and Schatz, G. (1991). Trends Cell Biol. 1, 99 103.

Hackenbrock, C. R. (1968). Proc. Natl. Acad. Sci. USA 61, 598-605. Hartl, F.-U., and Neupert, W. (1990). Science 247, 930-938.

Hwang, S., Jascur, T., Vestweber, D., Pon, L., and Schatz, G. (1989). J. Cell Biol. 109, 487-493.

Hwang, S. T., Wachter, C., and Schatz, G. (1991). J. Biol. Chem. 266 21083-21089.

Kellems, R. E., Allison, V. F., and Butow, R. A. (1975). J. Cell Biol. 65 1-14.

Kiebler, M., Pfaller, R., Söliner, T., Griffiths, G., Horstmann, H., Pfanner, N., and Neupert, W. (1990). Nature 348, 610-616.

Koronakis, V., Hughes, C., and Koronakis, E. (1991). EMBO J. 10. 3263-3272

Lill, R., Stuart, R. A., Drygas, M. E., Nargang, F. E., and Neupert, W. (1992). EMBO J. 11, 449-456.

Martin, J., Mahlke, K., and Pianner, N. (1991). J. Biol. Chem. 266, 18051-18057.

Pfanner, N., and Neupert, W. (1987). J. Biol. Chem. 262, 7528-7536.

Pugsley, A. P., Poquet, I., and Kornacker, M. G. (1991). Mol. Microbiol 5, 865-873

Rassow, J., and Pfanner, N. (1991). FEBS Lett. 293, 85-88.

Rassow, J., Guiard, B., Wienhues, U., Herzog, V., Hartl, F.-U., and Neupert, W. (1989). J. Cell Biol. 109, 1421-1428.

Schleyer, M., and Neupert, W. (1985). Cell 43, 339-350.

Schwaiger, M., Herzog, V., and Neupert, W. (1987). J. Cell Biol. 105, 235-246.

Sen, K., and Nikaido, H. (1990). Proc. Natl. Acad. Sci. USA 87, 743747.

Söllner, T., Griffiths, G., Pfaller, R., Pfanner, N., and Neupert, W. (1989). Cell 59, 1061-1070.

Söllner, T., Pfaller, R., Griffiths, G., Pfanner, N., and Neupert, W. (1990). Cell 62, 107-115.

Van Venetie, R., and Verkleij, A. J. (1982). Biochim. Biophys. Acta 692 , 397-405.

Vestweber, D., Brunner, J., Baker, A., and Schatz, G. (1989). Nature 341, 205-209. 